\title{
HIDROGEOLOGIA DA BACIA DE ONDINA, REGIÃO METROPOLITANA
}

\section{DE SALVADOR-BA}

\author{
Lucas de Queiroz Salles ${ }^{1}$; Thiago dos Santos Gonçalves²; Sérgio Augusto de Morais Nascimento³; Luiz \\ Rogério Bastos Leal ${ }^{4}$; Ricardo Galeno Fraga de Araújo Pereira ${ }^{5}$; Fabiane Ferreira Natividade doa Santos ${ }^{6}$
}

\begin{abstract}
Resumo - A bacia hidrográfica de Ondina está totalmente inserida na região metropolitana de Salvador-Ba. Limita-se a Oeste pela bacia Barra / Centenário, a Sul pelo Oceano Atlântico, a Norte e Leste pela Bacia do Lucaia. Esse trabalho objetiva caracterizar a hidrogeologia da região, a partir do estudo de: (i) índices de saturação; (ii) especiação química dos elementos dissolvidos em água; (iii) correlação de dados não paramétricos; e por fim (iv) classificação da água quanto a salinidade e segundo os diagramas Pipper e Stiff, a partir da coleta em campo de oito poços tubulares. Para a classificação das águas foi utilizado o software Qualigraf (2014) da Fundação Cearense de Meteorologia e Recursos Hídricos - FUNCENE, os cálculos de saturação mineral e especiação química foram elaborados com o suporte do PHREEQC - do USGS - Serviço Geológico dos Estados Unidos. O tratamento estatístico dos dados foi realizado com auxílio do software Excel 2013. As águas em estudo foram classificadas como mista cloretada (37,5\%), cálcica cloretada $(12,5 \%)$, sódica bicarbonatada (12,5\%), sódica mista (12,5\%) e mista mista (12,5\%), onde os oxidos e hidróxidos de ferro apresentaram os maiores índices de saturação. A elevada heterogeneidade dos dados sugere que as águas em estudo pertencem a três fácies hidroquímicas com características fisico-quimicas distintas.
\end{abstract}

\section{Palavras-Chave - Hidrogeologia; Bacia de Ondina; PHREQC.}

Abstract - The hydrographic basin Ondina is fully inserted in the metropolitan region of SalvadorBa. It is limited to the West Basin Barra / Centenário, the south by the Atlantic Ocean, the North and East by Basin Lucaia. This papper aims to characterize the hydrogeology of the region, from the study: (i) saturation levels; (ii) chemical speciation of elements dissolved in water; (iii) correlation nonparametric data; and finally (iv) classification as water salinity and according to Piper and Stiff diagrams. For the classification of water was used Qualigraf software (2014) Cearense of Meteorology and Water Resources Foundation - FUNCENE, the calculation of mineral saturation and chemical speciation were developed with the support PHREEQC - USGS - United States Geological Survey. The statistical treatment of the data was done with Excel software 2013. The waters in the study were classified as mixed chlorinated (37.5\%), chlorinated calcium (12.5\%), sodium bicarbonate (12.5\%), mixed sodium (12.5\%) and mixed mixed (12.5\%), the oxides and hydroxides of iron showed the highest levels of saturation. The high heterogeneity of the data suggests that the waters in the study belong to three hydrochemical facies with different physicochemical characteristics.

\footnotetext{
${ }^{1}$ Mestrando do Programa de Pós-Graduação em Geologia - Universidade Federal da Bahia, Núcleo de Estudos Hidrogeológicos e do Meio Ambiente

- Nehma. Rua Barão de Geremoabo, s/n Campus Universitário de Ondina, CEP: 40.170-020, fone: (71) 32838637. Lucassales2008@ gmail.com.

2 Mestrando do Programa de Pós-Graduação em Geologia - Universidade Federal da Bahia, Núcleo de Estudos Hidrogeológicos e do Meio Ambiente

- Nehma. Rua Barão de Geremoabo, s/n Campus Universitário de Ondina, CEP: 40.170-020, fone: (71) 32838637. T.gon@ outlock.com.

${ }^{3}$ Professor do Departamento de Geofísica. - Universidade Federal da Bahia, Núcleo de Estudos Hidrogeológicos e do Meio Ambiente - Nehma. Rua Barão de Geremoabo, s/n Campus Universitário de Ondina, CEP: 40.170-020, fone: (71) 32838637. sergiomn@ufba.br.

${ }^{4}$ Professor do Departamento de Geofísica. - Universidade Federal da Bahia, Núcleo de Estudos Hidrogeológicos e do Meio Ambiente - Nehma. Rua Barão de Geremoabo, s/n Campus Universitário de Ondina, CEP: 40.170-020, fone: (71) 32838637. 1rogerio@ufba.br.

${ }^{5}$ Professor do Departamento de Oceanografia. - Universidade Federal da Bahia, Núcleo de Estudos Hidrogeológicos e do Meio Ambiente - Nehma. Rua Barão de Geremoabo, s/n Campus Universitário de Ondina, CEP: 40.170-020, fone: (71) 32838637 \& TERRAQUATRO Geologia e Meio Ambiente Ltda. ricardo@ terraquatro.com.br.
} 
6 Aluna especial do Programa de Pós-Graduação em Geologia - Universidade Federal da Bahia, Rua Barão de Geremoabo, s/n Campus Universitário de Ondina, CEP: 40.170-020. Fabianesantidade82@gmail.com.

\section{Localização e Contexto Fisiográficos da Área Em Estudo}

A área de estudo está situada no bairro de Ondina no município de Salvador, limitada pelas

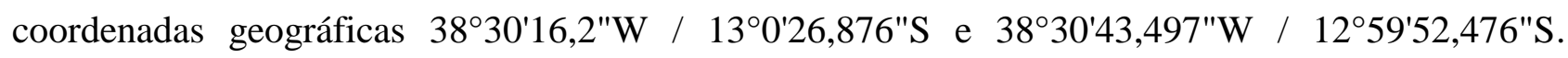
Apresenta-se predominantemente inserida nos limites do Campus de Ondina da Universidade Federal da Bahia, logo, apresenta fácil acesso, pelas avenidas Centenário e Anita Garibaldi.

O padrão de circulação das águas superficiais no perímetro urbano do município de Salvador sofreu intervenções ao longo dos últimos anos. Porém, ainda é possível identificar algumas bacias hidrográficas. Assim, a presente área em estudo encontra-se completamente inserida na sub bacia hidrográfica de Ondina (Figura 1). Sendo está sub bacia, limitada ao norte e a leste pela sub bacia do rio Lucaia, a oeste pela sub bacia hidrográfica do Comércio e a sul pelo Oceano Atlântico. De maneira geral, o padrão de circulação das águas superficiais na bacia hidrográfica de Ondina tem confluência para o Oceano Atlântico. 


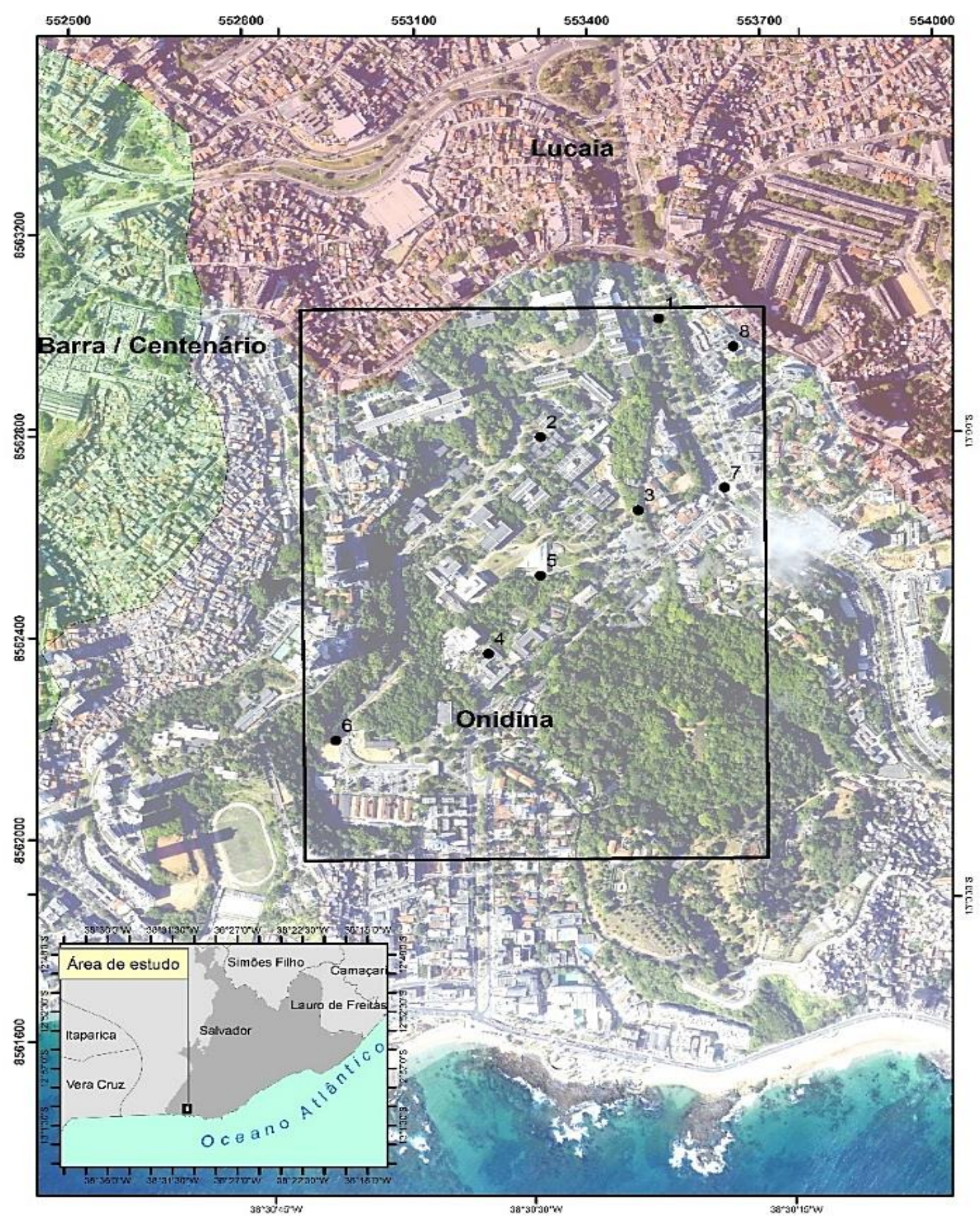

Figura 1: Sub bacias hidrográficas da região do bairro de Ondina. Em vermelho a sub bacia do rio Lucaia, em verde a sub bacia da Barra / Centenário e em branco a sub bacia de ondina. Detalhe para transparência nos polígonos.

Os aquíferos cristalinos da área em estudos estão instalados sobre rochas de composição predominantemente ácidas, com porções subordinadas básicas a ultrabásicas, denominadas por Complexo Salvador Explanada (SOUZA, 2013) (Figura 2). Todo sistema aquífero cristalino no município, está inserido nas rochas por meio de estruturas predominantemente rúpteis. 


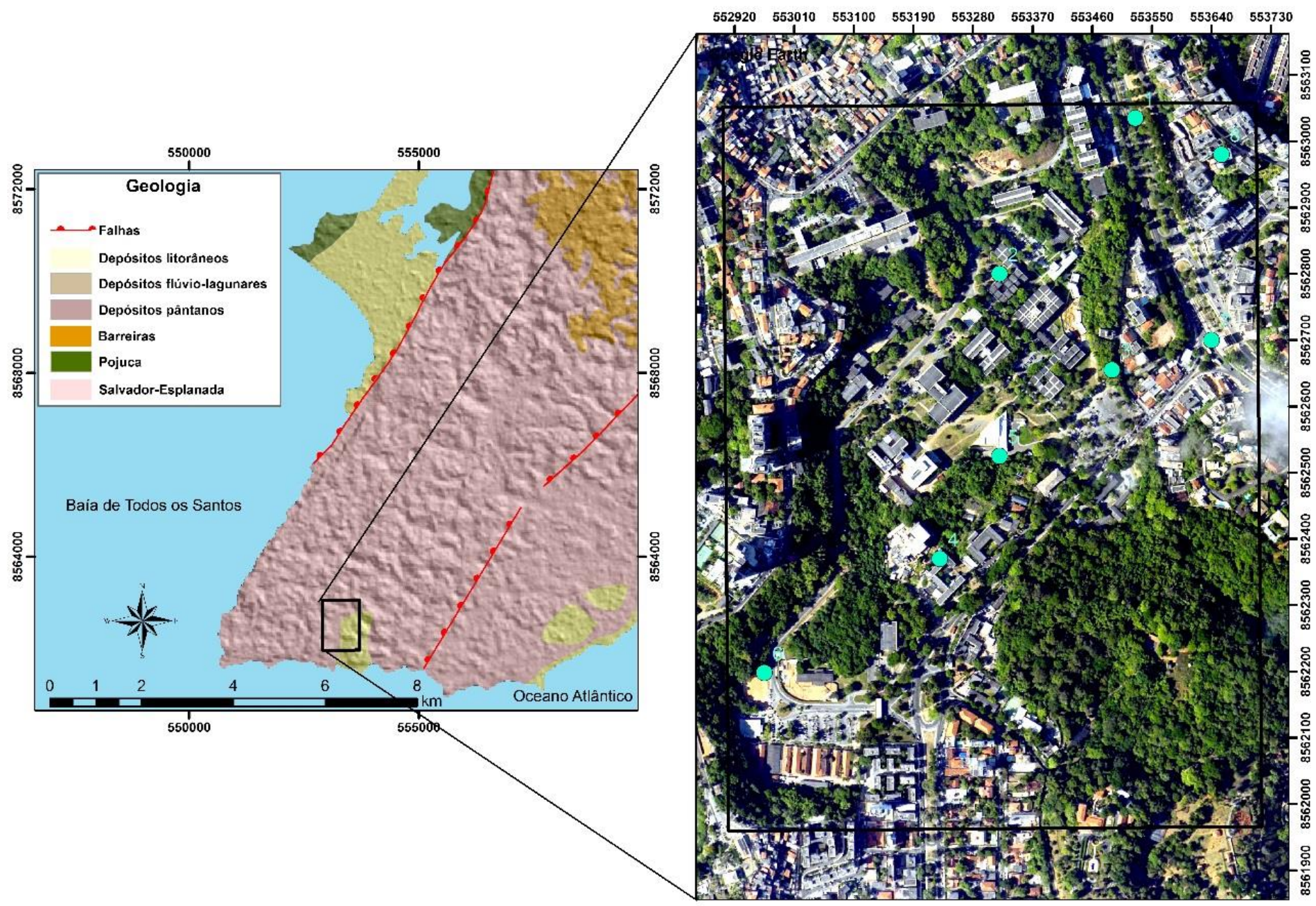

Figura 2: Mapa geológico de Salvador com detalhe para área em estudo. Fonte CPRM (2003).

Através da análise visual de imagens ASTERGDEM, foi possível extrair um total de 115 fotolineamentos. Sendo observados dois trends preferenciais N 300 e N00 (Figura 3), sistemas esses, compatíveis com os observados por Magnavita (1994). Assim, pode-se associar o padrão de armazenamento dos aquíferos fissurais do município de Salvador com os sistemas de falhas e fraturas da abertura da bacia do recôncavo. 

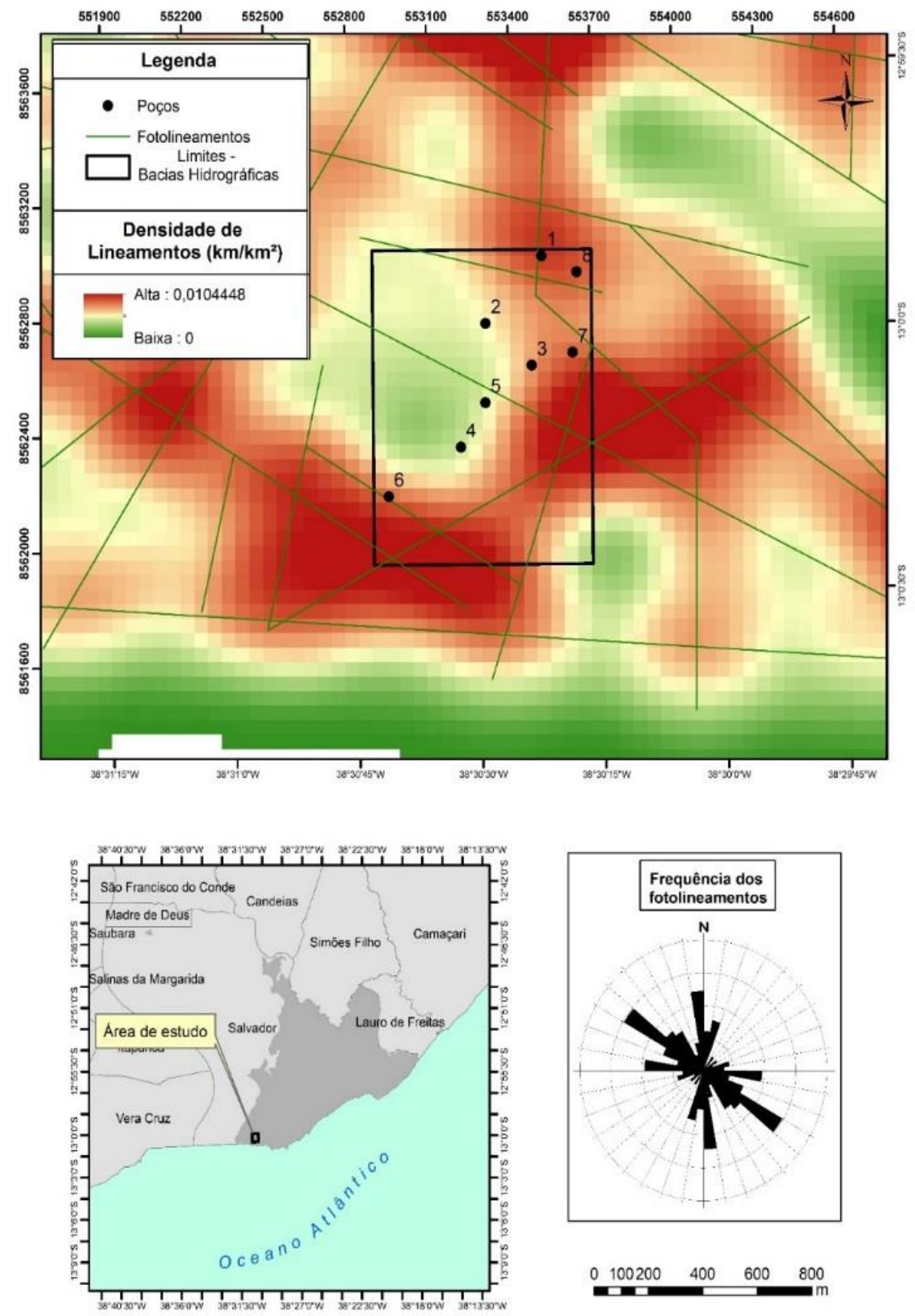

Figura 3: Mapa de isodensidade de fotolineamentos estrutruais para área de estudo. Acrescido pela roseta de frequência de lineamentos para o município de Salvador.

A região em estudo está inserida na unidade geomorfológica do Alto de Salvador, limitado a oeste pela escarpa da Falha de Salvador e a leste pela Falha do Iguatemi. Essa área é caracterizada por possuir altitudes variando de 22 metros a até 120 metros (Figura 4), com declividade moderada. As quebras do relevo são oriundas de paleodrenagens e antigas redes de escoamento superficiais, hoje em estágio avanço de antropização, tanto na forma de canalizações, como através da retificação 


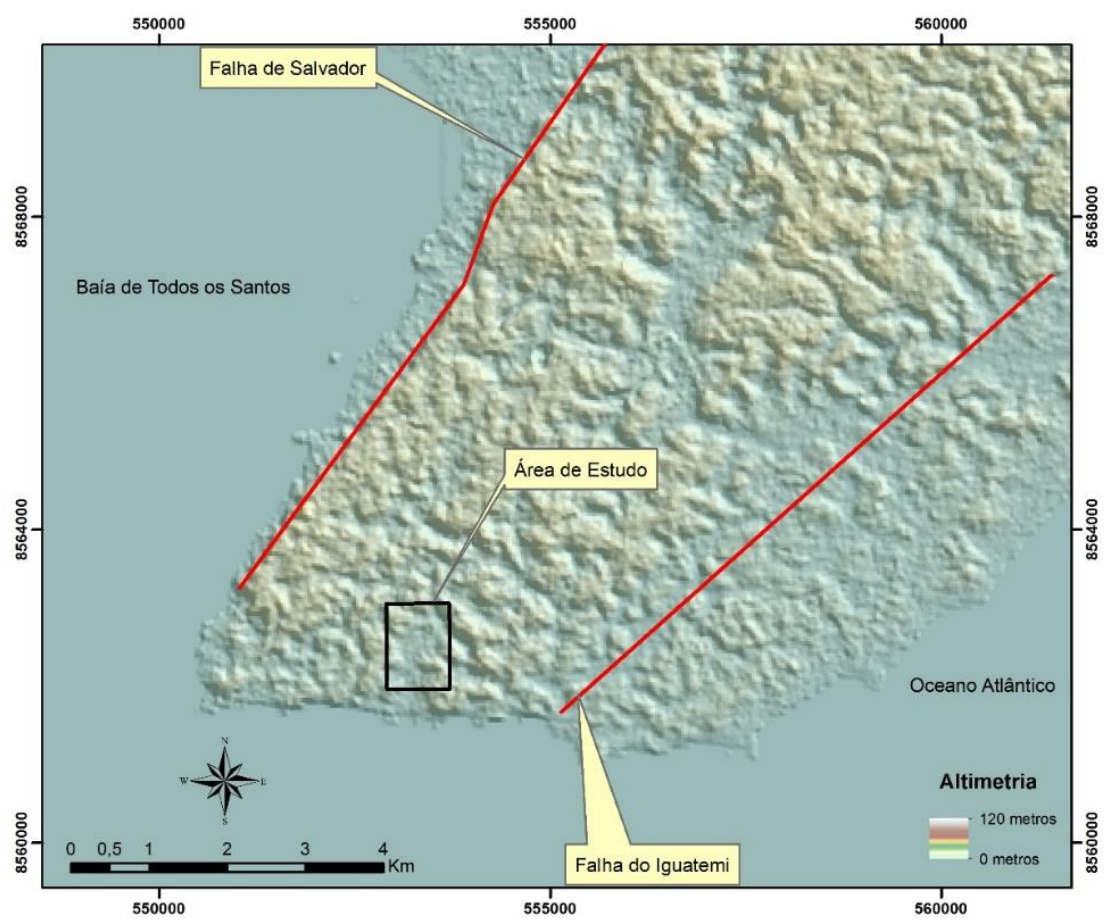

Figura 4: Modelo digital de elevação - MDT para a cidade de Salvador.

Quanto ao clima o município de Salvador é classificado como uma região tropical de baixas latitudes, o que permite a incidência de raios solares com praticamente a mesma intensidade durante todo o ano. Essas características propiciam elevadas temperaturas médias e pequenas amplitudes térmicas anuais, principalmente nas superfícies aplainadas.

Caracterizada por clima úmido, de relativa homogeneidade, o Município de Salvador, apresenta altos índices pluviométricos e médios térmicas elevados ao longo do ano. A temperatura média local varia entre $24^{\circ} \mathrm{C}$ e $30^{\circ} \mathrm{C}$, e a precipitação anual média fica entre 1100 e $2000 \mathrm{~mm}$ (Figura 5).

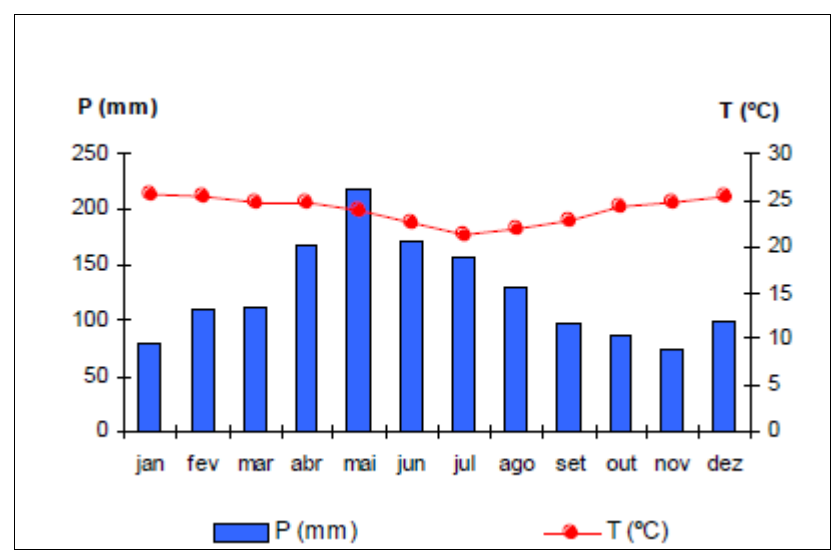

Figura 5: Variação da pluviometria e temperatura para o município de Salvador. 
A partir do cálculo de carga hidráulica foi possível sugerir o fluxo subterrâneo no aquífero fissural em estudo. Como pode-se perceber, na Figura 6, o fluxo apresenta-se convergente para a parte centro-sul da área em estudo, com sentido preferencial para sul, e altos piezométricos em sua porção nordeste.

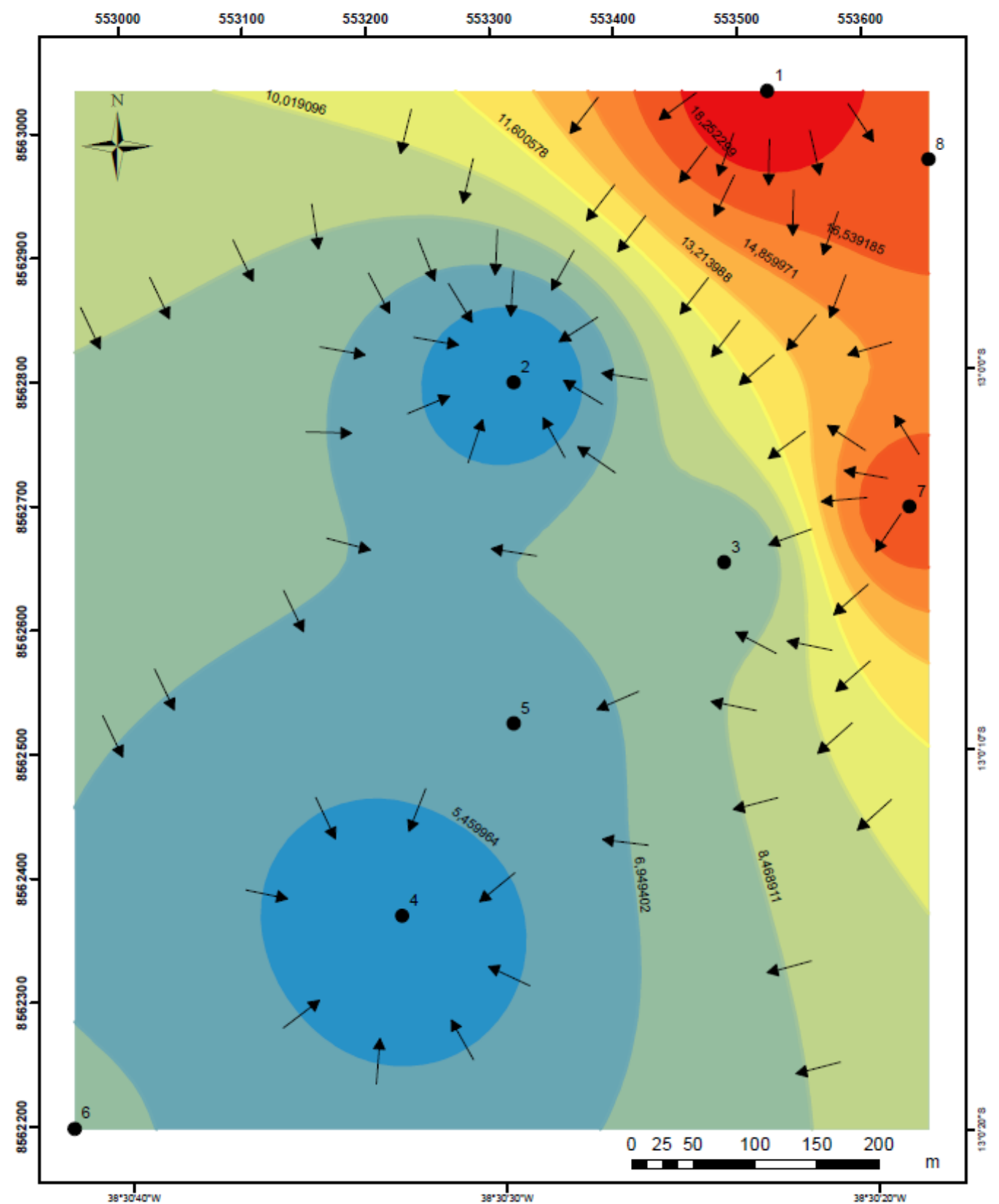

Figura 6: Mapa de fluxo subterrâneo para área de estudo, obtido a partir da espacialização da carga hidráulica medida em campo. 


\section{Classificação das Águas Subterrâneas (PIPER E STIFF)}

Para uma melhor compreensão dos resultados referentes à composição química das amostras analisadas, foram plotados os valores obtidos no diagrama triangular Piper, para classificação e comparação de distintos grupos de águas quanto aos íons dominantes e no diagrama Stiff, para representação gráfica do resultado de análise química da água quanto às características iônicas da mesma (Figura 7).

Os valores analíticos dos elementos maiores obtidos permitiram classificar as águas na região, segundo Piper (1994) como: mista cloretada (37,5\%), cálcica cloretada (12,5\%), sódica bicarbonatada (12,5\%), sódica mista $(12,5 \%)$ e mista mista (12,5\%) (Gráfico 1). Para auxiliar na classificação das águas mistas mista (Pto - 02), foi utilizado o diagrama de stiff (Figura 8), classificando-a como cloretada cálcica.

Gráfico 1: Distribuição das águas segundo Piper (1994).

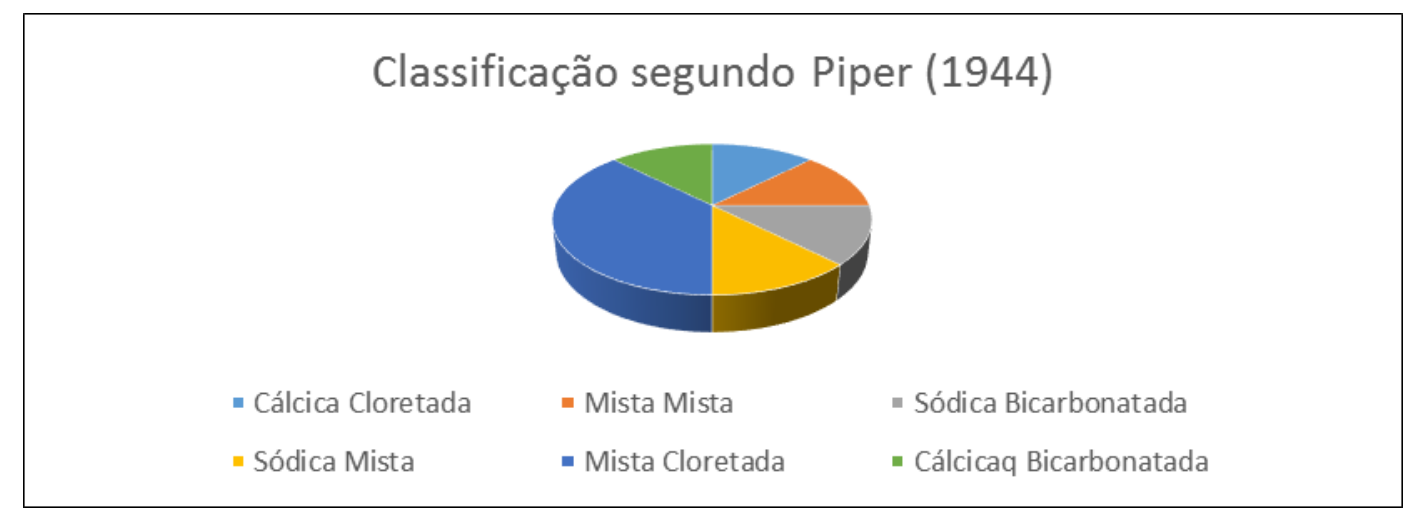




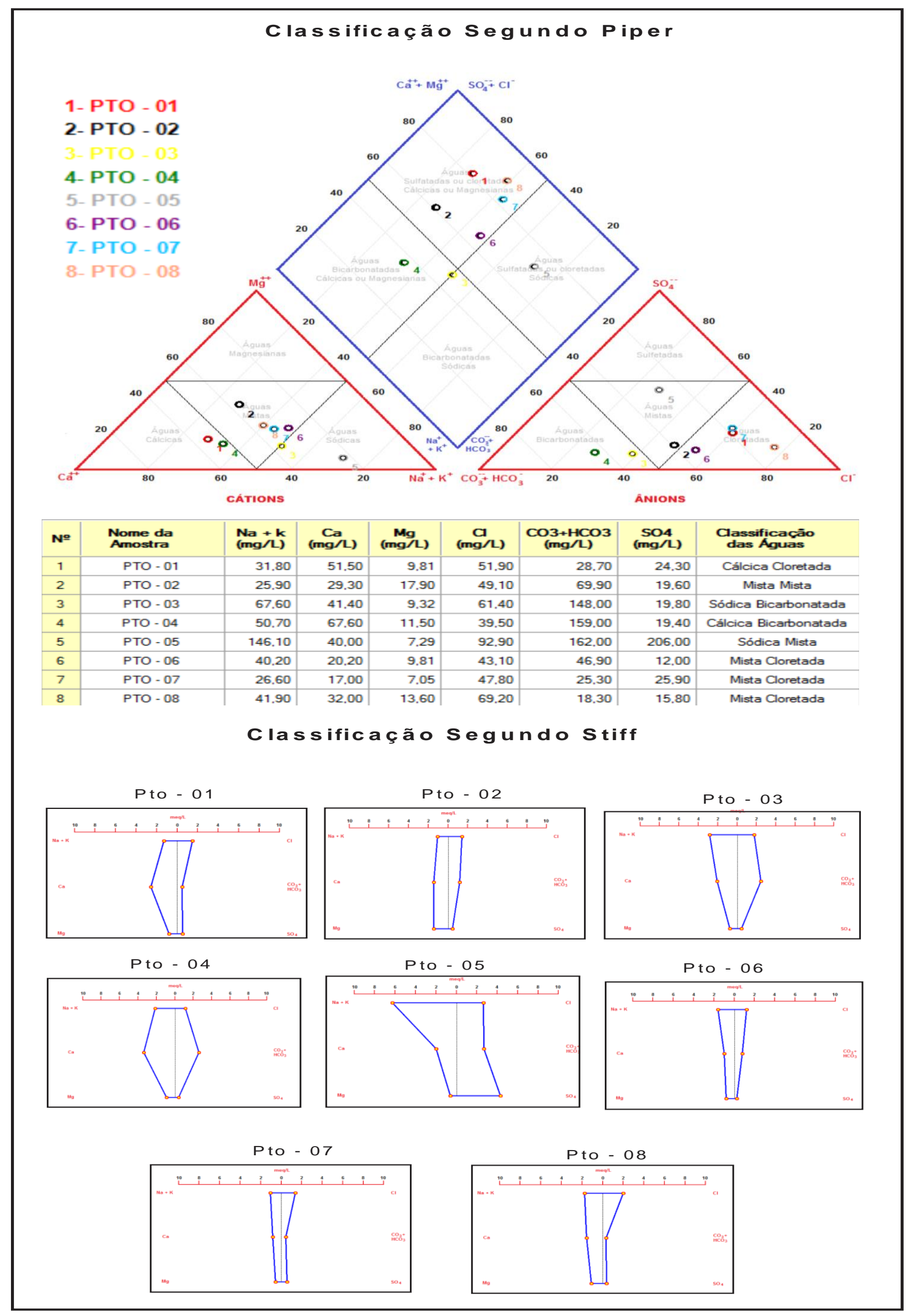

Figura 7: Classificação das águas em estudo segundo Piper e Stiff. Para as oito amostras estudadas. 


\section{STD - Salinidade das Águas Subterrâneas}

Os sólidos totais dissolvidos (STD) correspondem ao peso total dos constituintes inorgânicos e orgânicos adsorvidos por unidade de volume. Segundo a Resolução do Conselho Nacional do Meio Ambiente (CONAMA) 357/2005, com base no STD as águas podem ser divididas entre doces, salobras e salinas de acordo com as definições exemplificadas na tabela 1 abaixo.

Tabela 1: Classificação para salinidade segundo a Resolução do Conselho Nacional do Meio Ambiente (CONAMA) 357/2005.

CLASSIFICAÇÃO QUANTO A SALINIDADE

\section{Doce \\ Salobra \\ Salgada}

STD (MG/L)

$$
0-500
$$

$501-1500$

$>1500$

Segundo esta Resolução, as águas em estudo foram classificadas predominantemente como águas doces $(87,5 \%)$. Apenas o ponto 5 foi classificado como água salobra (12,5\%) (Figura 8).
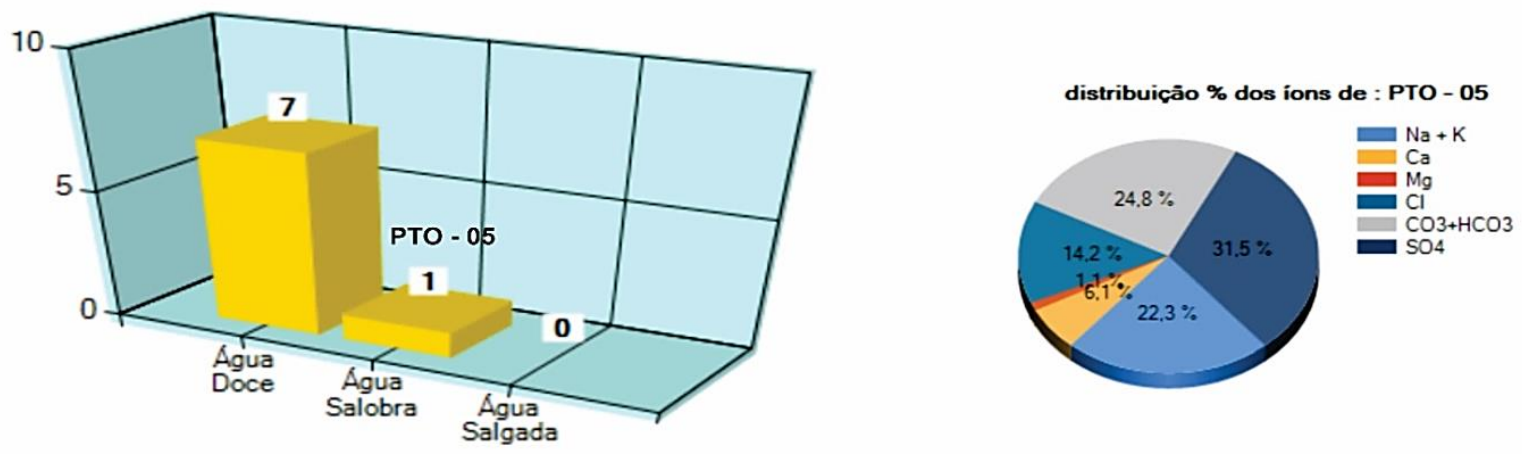

\begin{tabular}{|c|c|c|c|c|c|c|c|c|c|}
\hline Nome da Amostra & $\begin{array}{l}\mathrm{Na}+\mathrm{K} \\
(\mathrm{mg} / \mathrm{L})\end{array}$ & $\underset{(\mathrm{mg} / \mathrm{L})}{\mathrm{Ca}}$ & $\underset{(m g / L)}{M g}$ & $\underset{(m g / l)}{a}$ & $\begin{array}{c}\mathrm{CO3}+ \\
\mathrm{HCO} 3 \\
\text { (mg/L) }\end{array}$ & $\begin{array}{c}\mathrm{SO4} \\
(\mathrm{mg} / \mathrm{L})\end{array}$ & $\begin{array}{c}\text { C.E. } \\
(\mu \mathrm{S} / \mathrm{cm})\end{array}$ & $\begin{array}{c}\text { STD } \\
\text { estimado } \\
\text { (mg/l) }\end{array}$ & Classificaçāo \\
\hline PTO - 01 & 31.80 & 51.50 & 9.81 & 51.90 & 28.70 & 24.30 & 335 & 217.8 & Agua Doce \\
\hline PTO - 02 & 25.90 & 29.30 & 17.90 & 49.10 & 69.90 & 19.60 & 340 & 221.0 & Agua Doce \\
\hline PTO - 03 & 67.60 & 41.40 & 9.32 & 61.40 & 148.00 & 19.80 & 498 & 323.7 & Agua Doce \\
\hline PTO - 04 & 50.70 & 67.60 & 11.50 & 39.50 & 159.00 & 19.40 & 456 & 296.4 & Agua Doce \\
\hline РTO - 05 & 146.10 & 40.00 & 7.29 & 92,90 & 162.00 & 206.00 & 944 & 613.6 & Agua Salobra \\
\hline PTO - 06 & 40.20 & 20.20 & 9.81 & 43.10 & 46.90 & 12.00 & 275 & 178.8 & Agua Doce \\
\hline PTO - 07 & 26.60 & 17.00 & 7.05 & 47.80 & 25.30 & 25.90 & 217 & 141.1 & Agua Doce \\
\hline PTO - 08 & 41.90 & 32.00 & 13,60 & 69.20 & 18.30 & 15.80 & 368 & 239.2 & Agua Doce \\
\hline
\end{tabular}

Figura 8: Classificação das águas em estudo segundo a Resolução do Conselho Nacional do Meio Ambiente (CONAMA) 357/2005 para salinidade. 
Matriz de Correlação de Spearman e principais correlações positivas com a Condutividade Elétrica.

Com o intuito de verificar a correlação entre os elementos analisados nas águas subterrâneas em estudo foi utilizada a correlação de Spearman (Tabela 2), visto que esses dados não apresentam uma distribuição simétrica, inviabilizando uma correlação linear.

O coeficiente de correlação de Spearman $(\rho)$ é uma medida do grau de relação entre duas variáveis quantitativas. Este coeficiente varia entre os valores -1 e 1 . Se existir uma correlação direta entre os elementos análisados, $\rho$ estará próximo a 1. Se a correlação for inversamente proporcional, $\rho$ estará próximo a -1 . Se não há correlação ou uma correlação fraca, $\rho$ está próximo de zero. Adotou-se o valor $\geq 0,70$ para definir as correlações fortes entre os parâmetros analisados.

A partir da observação da matriz de correlação de Spearman identificou-se que o Potencial de Hidrogênio $(\mathrm{pH})$ apresenta forte correlação com o aumento de cálcio nas águas. A Condutividade Elétrica demonstrou forte correlação linear com o aumento de Sódio (Na) e o Bicarbonato (HCO3) (Figura 9).

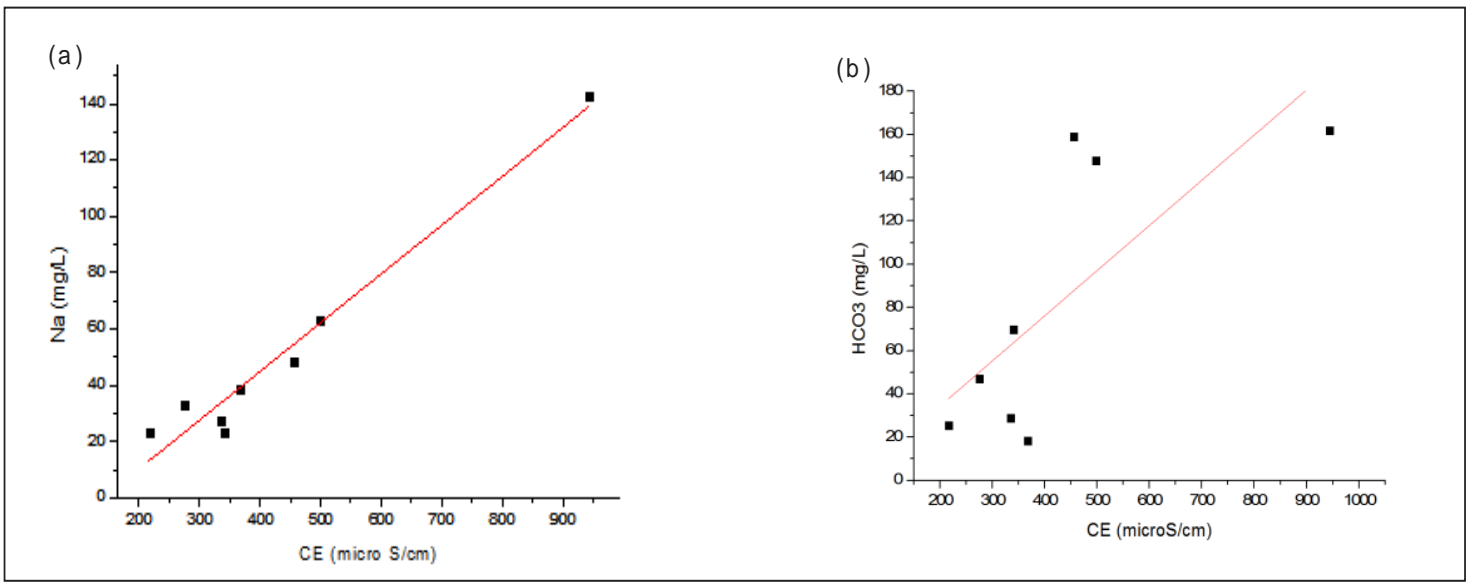

Figura 9: Correlação entre: (a) Condutividade Elétrica Vs Na; (b) Condutividade Elétrica Vs Bicarbonato.

Vale ressalvar ainda que houve forte correlação de Sperman entre: (i) a Cor e o ferro dissolvido, (ii) a dureza total e o magnésio, (iii) cloreto e cobre; e por fim (iv) entre o cromo e chumbo dissolvidos. 
Tabela 2: Matriz de correlação de Sperman para os 8 poços analisados.

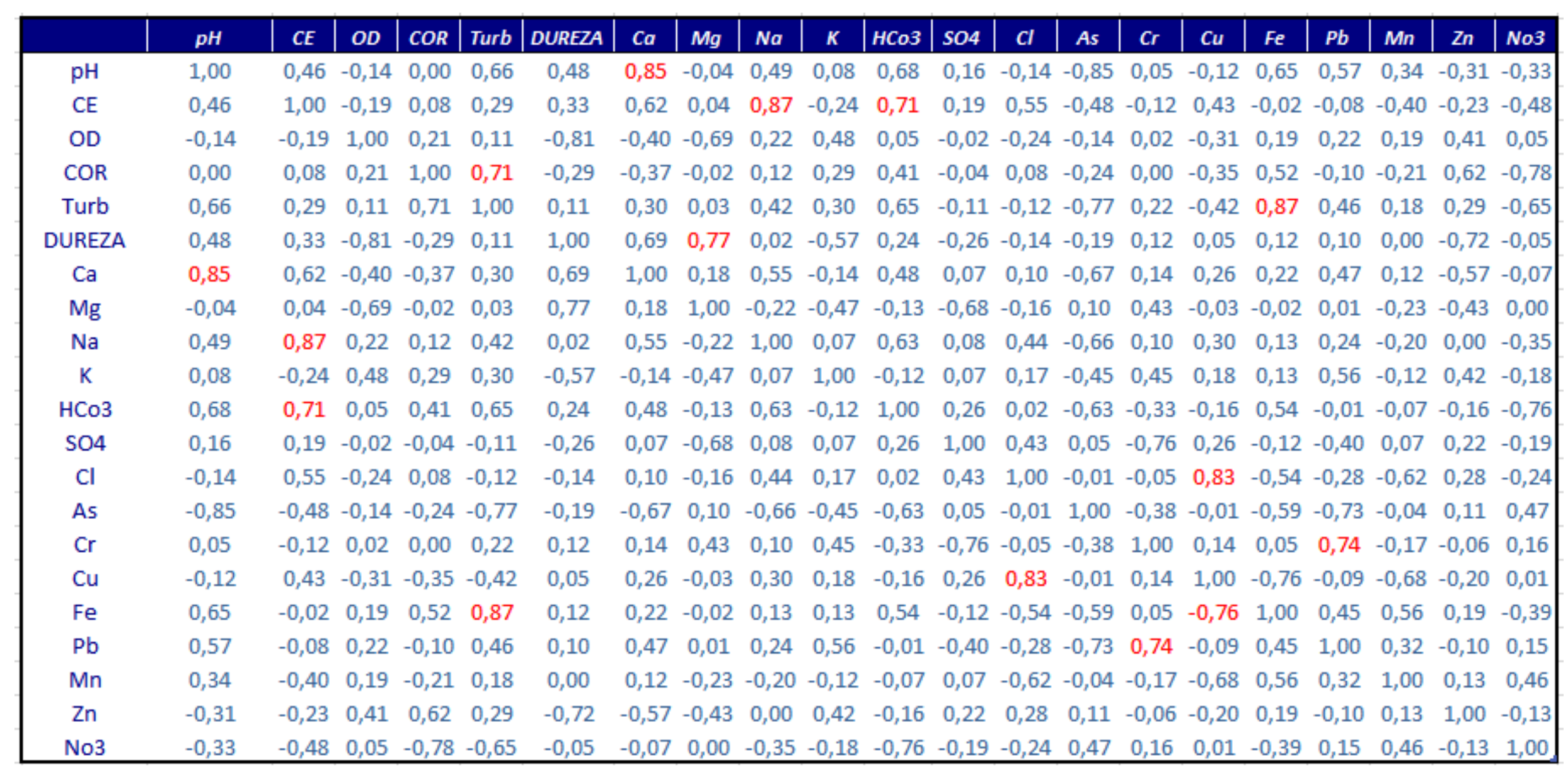




\section{Cálculo dos Índices de Saturação}

Para determinar as fases minerais, nas oito amostras coletadas, que se encontram nas águas subterrâneas do aquífero em estudo calculou-se o Índice de Saturação (IS) e as espécies que se encontram dissolvidas utilizando o software PHREEQE. Os elementos químicos utilizados no cálculo foram: DUREZA (mg/L), Ca (mg/L), Mg (mg/L), Na (mg/L), K (mg/L), HCo3 (mg/L), SO4 $(\mathrm{mg} / \mathrm{L}), \mathrm{Cl}(\mathrm{mg} / \mathrm{L}), \mathrm{As}(\mu \mathrm{g} / \mathrm{L}), \mathrm{Cr}(\mu \mathrm{g} / \mathrm{L}), \mathrm{Cu}(\mu \mathrm{g} / \mathrm{L}), \mathrm{Fe}(\mu \mathrm{g} / \mathrm{L}), \mathrm{Pb}(\mu \mathrm{g} / \mathrm{L}), \mathrm{Mn}(\mu \mathrm{g} / \mathrm{L}), \mathrm{Zn}(\mu \mathrm{g} / \mathrm{L})$, No3 (mg/L), também foi considerado o parâmetro $\mathrm{pH}$.

O índice de saturação (IS) indica se a solução está em equilíbrio, subsaturada ou supersaturada em relação a uma determinada fase sólida. Para área de estudo foram calculados os seguintes índices de saturação: anglesita $(\mathrm{PbSO} 4)$, anidrita $(\mathrm{CaSO} 4)$, aragonita $(\mathrm{CaCO} 3)$, calcita $(\mathrm{CaCO} 3)$, Cerrusita (PbCO3), $\quad \mathrm{CO} 2(\mathrm{~g}), \quad$ dolomita $(\mathrm{CaMg}(\mathrm{CO} 3) 2), \quad \mathrm{Fe}(\mathrm{OH}) 3$, goethita $(\mathrm{FeO}(\mathrm{OH}))$, Gipso (CaSO4.2H2O), H2(g), H2O(g), Halita (HCL), Hausmannita (Mn2+Mn3+2O4), Hematita (Fe2O3), Jarosita $\quad(\mathrm{K} 2 \mathrm{Fe} 6(\mathrm{OH}) 12(\mathrm{SO} 4) 4)$, Manganita $\quad(\mathrm{MnO}(\mathrm{OH}))$, Melanterita $(\mathrm{FeSO} 4 \cdot 7 \mathrm{H} 2 \mathrm{O}), \mathrm{O} 2(\mathrm{~g})$ ,Pb(OH)2, Pyrochroite $(\mathrm{Mn}(\mathrm{OH}) 2)$, Pirolusita (MnO2), Rhodochrosite (MnCO3), Siderita (FeCO3), Smithsonite, Silvania $(\mathrm{KCl})$ e $\mathrm{Zn}(\mathrm{OH}) 2$, exemplificados na tabela 3.

Tabela 3: Índices de saturação calculados.

\begin{tabular}{|c|c|c|c|c|c|c|c|c|}
\hline Faes Minerais & PTO-01 & PTO-02 & PTO-03 & PTO-04 & PTO-05 & PTO-06 & PTO-07 & PTO-08 \\
\hline Anglesita (PbSO4) & -2.34 & -5.08 & -3.15 & -3.98 & -3.75 & -2.57 & -4.57 & -3.81 \\
\hline Anidrita (CaSO4) & -2.57 & -2.89 & -2.77 & -2.60 & -1.89 & -3.22 & -2.95 & -2.94 \\
\hline Aragonita (CaCO3) & -2.02 & -2.37 & -1.62 & 0.00 & -1.59 & -2.58 & -3.12 & -3.00 \\
\hline Calcita $(\mathrm{CaCO} 3)$ & -1.88 & -2.22 & -1.47 & 0.14 & -1.45 & -2.44 & -2.98 & -2.86 \\
\hline Cerrusita (PbCO3) & -0.50 & -3.28 & -0.71 & -0.09 & -2.17 & -0.66 & -3.46 & -2.60 \\
\hline $\mathrm{CO} 2(\mathrm{~g})$ & -1.77 & -0.88 & -0.86 & -2.24 & -0.93 & -1.15 & -1.21 & -1.36 \\
\hline Dolomita (CaMg(CO3)2) & -4.13 & -4.31 & -3.25 & -0.13 & -3.31 & -4.84 & -5.99 & -5.75 \\
\hline $\mathrm{Fe}(\mathrm{OH}) 3(\mathrm{a})$ & 0.31 & -1.21 & -0.93 & 3.30 & 0.14 & -0.55 & -1.84 & -2.08 \\
\hline Goethita $(\mathrm{FeO}(\mathrm{OH}))$ & 6.20 & 4.68 & 4.96 & 9.19 & 6.03 & 5.34 & 4.05 & 3.81 \\
\hline Gipso (CaSO4.2H2O) & -2.27 & -2.59 & -2.46 & -2.30 & -1.58 & -2.91 & -2.64 & -2.64 \\
\hline $\mathrm{H} 2(\mathrm{~g})$ & -20.65 & -19.65 & -20.25 & -23.05 & -20.45 & -19.85 & -19.45 & -19.45 \\
\hline $\mathrm{H} 2 \mathrm{O}(\mathrm{g})$ & -1.50 & -1.50 & -1.50 & -1.50 & -1.50 & -1.50 & -1.50 & -1.50 \\
\hline Halita (HCL) & -7.13 & -7.49 & -6.97 & -7.28 & -6.46 & -7.39 & -7.50 & -7.18 \\
\hline Hausmannita (Mn2+Mn3+2O4) & -17.57 & -24.58 & -24.02 & -7.75 & -20.32 & -20.75 & -21.79 & -24.59 \\
\hline Hematita (Fe2O3) & 14.41 & 11.37 & 11.93 & 20.39 & 14.07 & 12.69 & 10.10 & 9.62 \\
\hline Jarosita (K2Fe6(OH)12(SO4)4) & -5.68 & -9.11 & -9.01 & -0.81 & -4.15 & -7.37 & -10.27 & -11.55 \\
\hline Manganita $(\mathrm{MnO}(\mathrm{OH}))$ & -7.42 & -9.92 & -9.64 & -3.75 & -8.37 & -8.61 & -9.03 & -9.96 \\
\hline Melanterita (FeSO4-7H2O) & -6.28 & -6.38 & -7.01 & -7.01 & -5.25 & -6.20 & -6.55 & -7.04 \\
\hline $\mathrm{O} 2(\mathrm{~g})$ & -41.99 & -43.99 & -42.79 & -37.19 & -42.39 & -43.59 & -44.39 & -44.39 \\
\hline $\mathrm{Pb}(\mathrm{OH}) 2$ & -1.87 & -5.53 & -2.99 & -0.99 & -4.38 & -2.64 & -5.39 & -4.37 \\
\hline Pyrochroite $(\mathrm{Mn}(\mathrm{OH}) 2)$ & -7.58 & -9.58 & -9.60 & -5.11 & -8.43 & -8.37 & -8.59 & -9.52 \\
\hline Pirolusita (MnO2) & -13.16 & -16.16 & -15.58 & -8.29 & -14.21 & -14.75 & -15.37 & -16.30 \\
\hline Rhodochrosite (MnCO3) & -1.16 & -2.28 & -2.27 & 0.84 & -1.17 & -1.34 & -1.61 & -2.69 \\
\hline Siderita (FeCO3) & -1.10 & -1.23 & -1.23 & 0.22 & -0.33 & -0.94 & -2.10 & -2.49 \\
\hline Smithsonite & -3.95 & -4.08 & -3.86 & -2.63 & -2.02 & -3.44 & -4.38 & -4.56 \\
\hline Silvania $(\mathrm{KCl})$ & -7.75 & -7.97 & -7.71 & -8.13 & -7.56 & -7.61 & -7.88 & -7.83 \\
\hline $\mathrm{Zn}(\mathrm{OH}) 2(\mathrm{e})$ & -5.53 & -6.56 & -6.36 & -3.74 & -4.44 & -5.65 & -6.53 & -6.56 \\
\hline
\end{tabular}


Para melhor entendimento da área de estudo foi confeccionado um box plot para as fases minerais que contém o íon Fe em sua composição (Figura 12). Os índices de saturação em $\mathrm{Fe}(\mathrm{OH})_{3}$ variaram entre -1,21 a 0,31; os índices de saturação em Goethita variam de 3,81 a 9,19; por fim, o índice de saturação em Hematita variou entre 9,62 e 20,39.

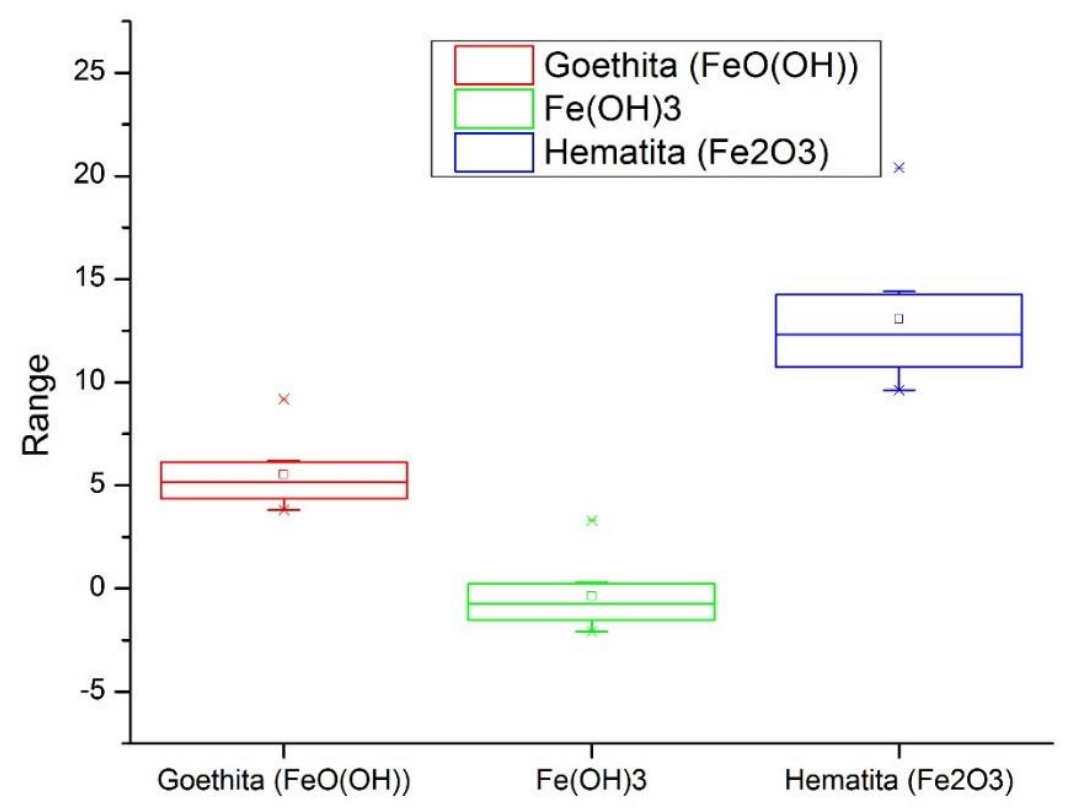

Figura 52: Box plot para os índices de saturação em Goethita, $\mathrm{Fe}(\mathrm{OH}) 3$ e Hematita.

\section{Especiação Química dos Elementos}

Especiação química consiste na distribuição de um dado elemento entre as espécies químicas de um sistema. Esse estudo se faz necessário em virtude de que as distribuições das espécies desses elementos podem afetar diretamente em suas características taxológicas. Vale ressaltar também, que as mobilidades dos íons metálicos estão diretamente ligadas à sua especiação. As espécies químicas para os elementos em estudos estão exemplificadas na tabela 4. 
Tabela 4: Espécies químicas para os elementos em estudos, obtidos a partir do phreeqc.

\begin{tabular}{|c|c|}
\hline Elemento & Espécie Química \\
\hline $\mathrm{C}(4)$ & $\mathrm{CO} 2 ; \mathrm{HCO}-; \mathrm{CaHCO}_{+}+$ \\
\hline $\mathrm{Ca}$ & $\mathrm{Ca}+2$ \\
\hline $\mathrm{Cl}$ & $\mathrm{Cl}-$ \\
\hline $\mathrm{Cu}$ & $\mathrm{Cu}+; \mathrm{CuCl} 2-; \mathrm{CuHCO}+; \mathrm{CuCO} 3$ \\
\hline $\mathrm{Fe}$ & $\mathrm{Fe}+2 ; \mathrm{FeHCO}+; \mathrm{FeSO} 4$ \\
\hline $\mathrm{H}$ & $\mathrm{H} 2$ \\
\hline $\mathrm{Mg}+2$ \\
\hline $\mathrm{Mn}$ & $\mathrm{Mn}+2 ; \mathrm{MnHCO} ; \mathrm{Mn}+3$ \\
\hline $\mathrm{N}$ & $\mathrm{NO} 3$ \\
\hline $\mathrm{Na}$ & $\mathrm{Na}+$ \\
\hline $\mathrm{O}$ & $\mathrm{O} 2$ \\
\hline $\mathrm{Pb}$ & $\mathrm{PbCO} 3 ; \mathrm{PbHCO}+; \mathrm{Pb}+2$ \\
\hline $\mathrm{S}$ & $\mathrm{SO} 4-2$ \\
\hline $\mathrm{Zn}$ & $\mathrm{Zn}+2$ \\
\hline
\end{tabular}

\section{Conclusões}

O estudo hidroquímico realizado na Bacia de Ondina eve como base atributos e elementos do meio físico local, em especial, a geologia, caracterização do solo e das águas subterrâneas, permitindo assim as seguintes conclusões:

I. As águas subterrâneas do aquífero fissural em estudo apresentam características hidroquímicas típicas de águas com moderado grau de mineralização. De acordo com o Diagrama Triangular de Piper, foram classificadas como mista cloretada (37,5\%), cálcica cloretada $(12,5 \%)$, sódica bicarbonatada $(12,5 \%)$, sódica mista $(12,5 \%)$ e sódica mista $(12,5 \%)$;

II. As águas em estudo foram classificadas predominantemente como águas doce $(87,5 \%)$. Apenas o ponto 5 foi classificado como água salobra, sugerindo diferentes fácies hidroquímicas $12,5 \%$;

III. A elevada heterogeneidade dos dados sugere que as águas em estudo são pertencentes a fácies hidroquímicas distintas. Segundo Mestrinho (2008) a condutividade elétrica e os 
índices de saturação devem apresentar uma evolução nas águas subterrânea. Daí uma correlação entre esses dois fatores devem agrupar fácies hidroquímicas distintas (Figura 13).

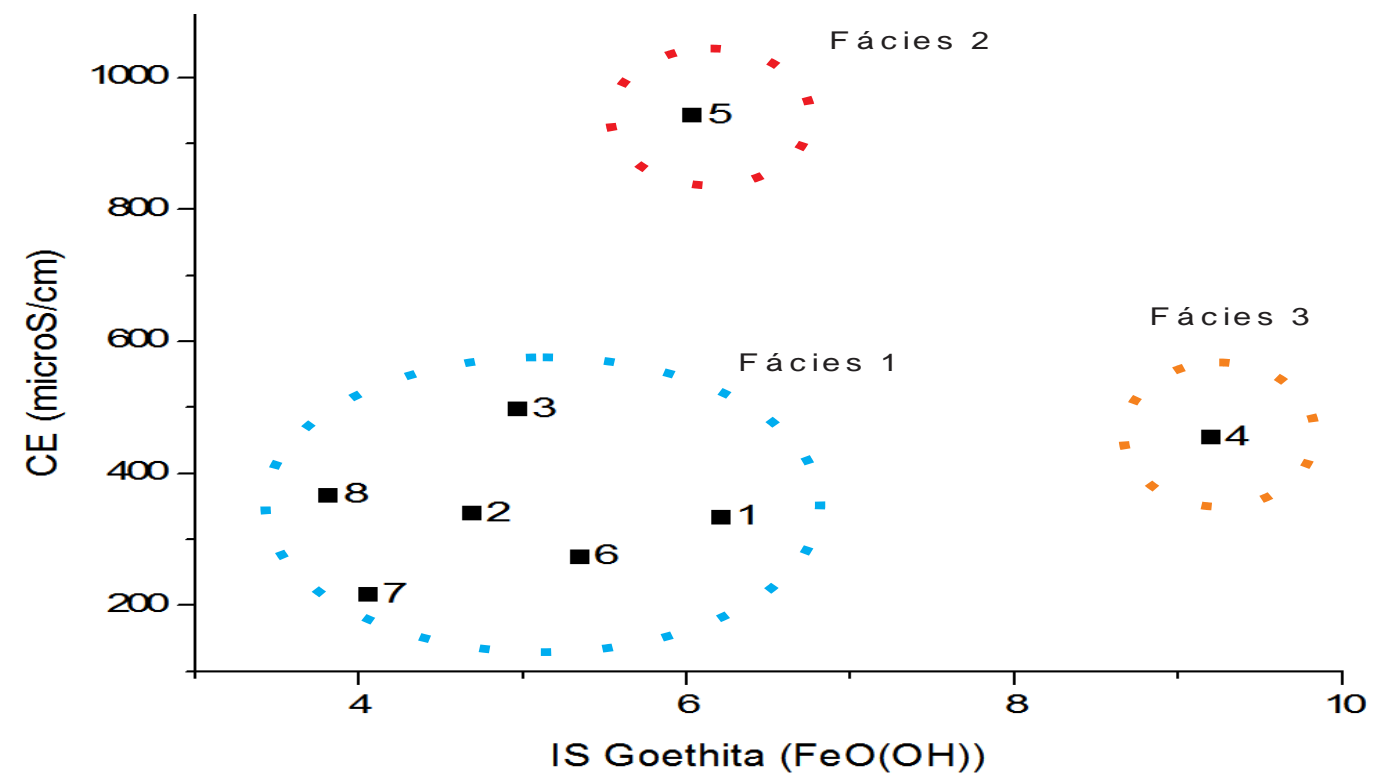

Figura 13: CE vs IS em Goethita como ferramenta para separar as fácies hidroquímicas.

A partir da correlação entre condutividade elétrica e o índice de saturação em Goethita foi possível delimitar três fácies hidroquímicas distintas para as águas em estudos. A primeira com baixos valores de condutividade elétrica e baixo índice de saturação em Goethita (ISG), a segunda apresenta elevada condutividade elétrica e moderado ISG; por fim a terceira fácies hidroquímica apresenta baixa condutividade e elevados ISG.

\section{REFERÊNCIAS}

BRASIL. Resolução CONAMA No 356/2004 do Conselho Nacional do Meio Ambiente. Prorroga o prazo estabelecido no art. 15 da Resolução CONAMA no 289, de 25 de outubro de 2001, que estabelece diretrizes para o Licenciamento Ambiental de Projetos de Assentamentos de Reforma Agrária". Ministério do Meio Ambiente, Brasília, 2004. pág. 082

BRASIL. RESOLUÇÃO CONAMA No 396/2008 do Conselho Nacional do Meio Ambiente. Dispõe sobre a classificação e diretrizes ambientais para o enquadramento das águas subterrâneas e dá outras providências. Brasil, 2008. 
BRASIL. Portaria $n^{\circ}$ 2914, de 12 dezembro de 2011. Normas de qualidade da água para consumo humano. Ministério da Saúde, Brasília, 2011. 32p.

HOLANDA, J. S. de \& AMORIM, J. R. A. de. Qualidade da água para irrigação. In: GHEYI, H. R.; QUEIROZ, J. E.; MEDEIROS, J. F. de. (ed.). Manejo e controle da salinidade na agricultura irrigada. Campina Grande: UFPB, 1997, p.137-169.

MESTRINHO; S. S. P. Geoquímica de Águas Subterrâneas. Livro Hidrogeologia Conceitos e Aplicações $3^{\circ}$ edição - Revisada e Ampliada. Capitulo 5.2, págs. 359-372. 2008 